\title{
funRNA: a fungi-centered genomics platform for genes encoding key components of RNAi
}

\author{
Jaeyoung Choi ${ }^{1,2}$, Ki-Tae Kim², Jongbum Jeon ${ }^{2}$, Jiayao $\mathrm{Wu}^{3}$, Hyeunjeong Song ${ }^{2}$, Fred O Asiegbu ${ }^{3}$, \\ Yong-Hwan Lee ${ }^{1,2,3,4^{*}}$ \\ From Asia Pacific Bioinformatics Network (APBioNet) Thirteenth International Conference on Bioinformatics \\ (InCoB2014) \\ Sydney, Australia. 31 July - 2 August 2014
}

\begin{abstract}
Background: RNA interference (RNAi) is involved in genome defense as well as diverse cellular, developmental, and physiological processes. Key components of RNAi are Argonaute, Dicer, and RNA-dependent RNA polymerase (RdRP), which have been functionally characterized mainly in model organisms. The key components are believed to exist throughout eukaryotes; however, there is no systematic platform for archiving and dissecting these important gene families. In addition, few fungi have been studied to date, limiting our understanding of RNAi in fungi. Here we present funRNA http://funrna.riceblast.snu.ac.kr/, a fungal kingdom-wide comparative genomics platform for putative genes encoding Argonaute, Dicer, and RdRP.

Description: To identify and archive genes encoding the abovementioned key components, protein domain profiles were determined from reference sequences obtained from UniProtKB/SwissProt. The domain profiles were searched using fungal, metazoan, and plant genomes, as well as bacterial and archaeal genomes. 1,163, 442, and 678 genes encoding Argonaute, Dicer, and RdRP, respectively, were predicted. Based on the identification results, active site variation of Argonaute, diversification of Dicer, and sequence analysis of RdRP were discussed in a fungus-oriented manner. funRNA provides results from diverse bioinformatics programs and job submission forms for BLAST, BLASTMatrix, and ClustalW. Furthermore, sequence collections created in funRNA are synced with several gene family analysis portals and databases, offering further analysis opportunities.

Conclusions: funRNA provides identification results from a broad taxonomic range and diverse analysis functions, and could be used in diverse comparative and evolutionary studies. It could serve as a versatile genomics workbench for key components of RNAi.
\end{abstract}

\section{Background}

RNA interference (RNAi), a term first coined in research on Caenorhabditis elegans, was originally thought to be a host defense mechanism against invasion of viral genomes or transposable elements [1]. However, several molecular studies revealed that it is also involved in diverse cellular, developmental, and physiological processes [2-5]. Gene silencing by RNAi begins with recognition of aberrant RNA (aRNA) or introduction of double-stranded RNA

\footnotetext{
* Correspondence: yonglee@snu.ac.kr

'Center for Fungal Pathogenesis, Seoul National University, Seoul 151-921, Korea

Full list of author information is available at the end of the article
}

(dsRNA), such as viral genomes. RNA-dependent RNA polymerase (RdRP) is responsible for the generation of dsRNA from aRNA. Dicer slices dsRNA into small (21-25 nt) pieces. Argonaute then acts on these fragments by forming an RNA-induced silencing complex (RISC), which is subsequently guided to target mRNAs, resulting in gene silencing.

In fungi, molecular characterization of genes encoding RNAi components has been intensively studied in Neurospora crassa and Schizosaccharomyces pombe. In N. crassa, there are two characterized post-transcriptional gene silencing (PTGS) mechanisms: quelling in a vegetative state and meiotic silencing by unpaired DNA (MSUD) at the 
sexual stage. Inspired by transcriptional down-regulation in albino-1 (al-1) or albino-3 (al-3) gene-overexpressing strains of $N$. crassa [6-8], quelling-defective (qde) mutants including qde-1, qde-2, and $q d e-3$, as well as the Dicerencoding genes $d c l-1$ and $d c l-2$, were characterized [9-12]. Differences between the two pathways include the occurrence of MSUD during prophase in meiosis I and the proteins involved in the pathways. In quelling, RdRP QDE-1 and Argonaute QDE-2 are required, whereas MSUD utilizes their paralogs, SAD-1 (suppressor of ascus dominance 1) and SMS-2 (suppressor of meiotic silencing 2), respectively $[13,14]$. In filamentous fungi, such as the abovementioned $N$. crassa, gene silencing mediated by RNAi occurs post-transcriptionally. On the other hand, in $S$. pombe, RNAi contributes to transcriptional gene silencing through heterochromatin formation [15].

Despite the importance of this universal machinery in eukaryotes, many studies on RNAi focused only on functional, physiological, and molecular aspects, rather than comparative genomics. It is known that particular fungal taxa, for example budding yeasts, do not have the key components of RNAi [16]. Hence a systematic, extensive identification and evolution analysis are needed to determine the clear distribution of the genes and to trace their evolutionary histories. Furthermore, considering that Argonaute-encoding genes were found in a few noneukaryotic species [17], the taxonomic distribution and phyletic trajectory of these important genes could tell us more about their ancestral origin. As a solution, we developed funRNA http://funrna.riceblast.snu.ac.kr/ to provide a gene catalogue based on 1,440 genomes and a comparative, evolutionary genomics platform for the genes encoding Argonaute, Dicer, and RdRP.

In this paper, we discuss the following: i) the taxonomic distribution of the key components of RNAi; ii) sequence analysis of predicted RdRPs by multiple sequence alignment; iii) auxiliary domain variation in Dicers; iv) evolutionary analysis of the putative genes encoding Argonautes by gene duplication and loss; and v) database and web functionalities available on the funRNA website.

\section{Results and discussion}

\section{Content and distribution of the identified genes encoding} Argonaute, Dicer, and RdRP

In order to predict putative genes, 1,440 genomes were searched using protein domain profiles (Figure 1; see Methods for details). 1,163 Argonaute-encoding genes, 442 Dicer-encoding genes, and 678 RdRP-encoding genes were predicted (Additional file 1). In order to evaluate the accuracy of the pipeline, a test set was prepared by retrieving sequences annotated as Argonaute, Dicer, and RdRP from UniProtKB/TrEMBL [18]. Assuming that the annotation provided by UniProtKB/TrEMBL is correct, the funRNA pipeline correctly captured $93.50 \%$ of the test set.
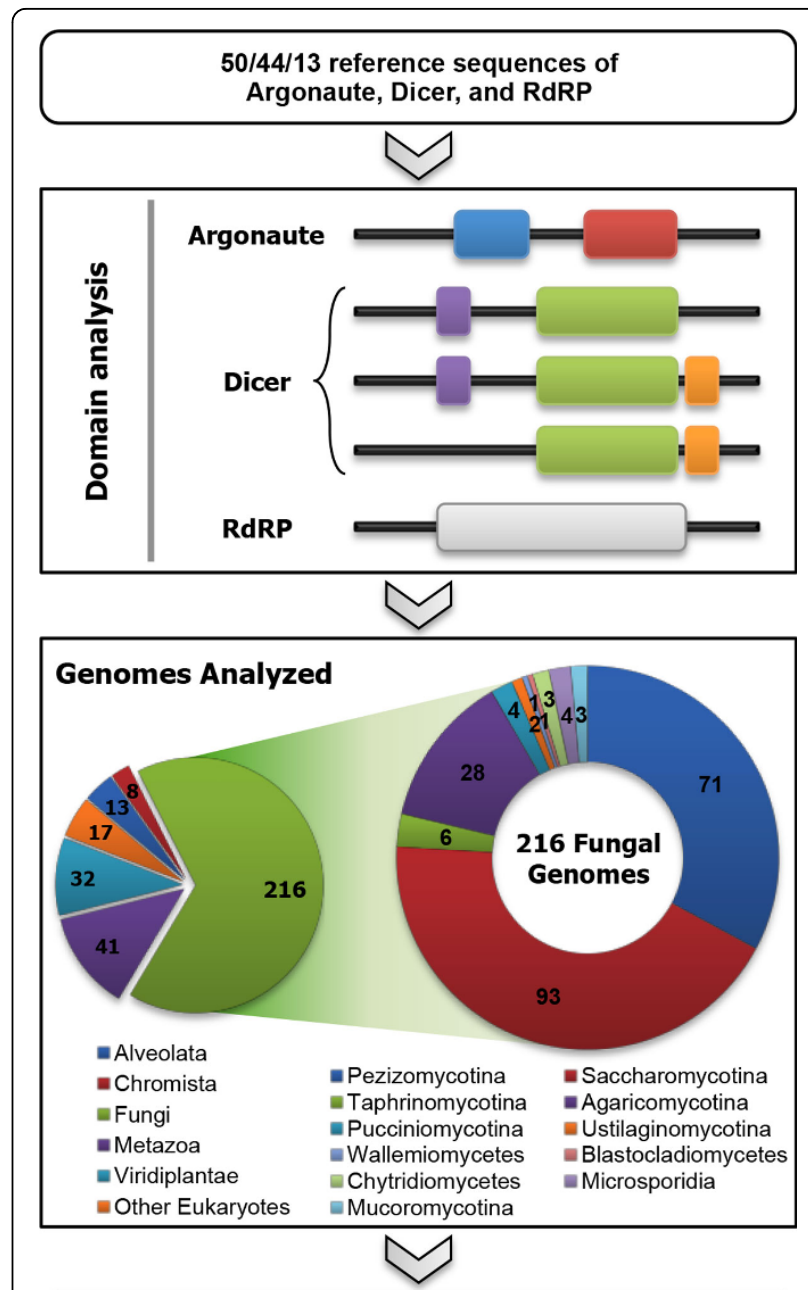

In total of 1,163, 442, and 678 putative genes encoding Arg onaute, Dicer, and RdRP, respectively, were predicted.

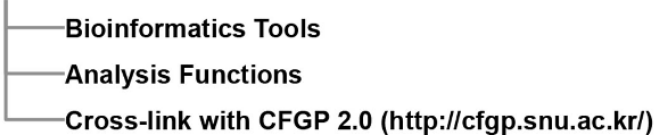

Figure 1 Identification pipeline for funRNA. The identification pipeline for funRNA consists of two steps: i) defining domain profiles from protein sequences encoded by the reference sequences; and ii) scanning 1,440 proteomes with domain profiles for Argonaute, Dicer, and RdRP. In "Domain analysis", colored boxes indicate essential domains: blue, IPR003100 (Argonaute/Dicer protein, PAZ); red, IPR003165 (Stem cell self-renewal protein Piwi); purple, IPR005034 (Dicer double-stranded RNA-binding fold); green, IPR000999 (Ribonuclease III); orange, IPR001159 (Double-stranded RNA-binding); and gray, IPR007855 (RNA-dependent RNA polymerase, eukaryotic type). In addition, sequences collected from funRNA can be subjected to bioinformatics analysis on the funRNA website as well as in CFGP 2.0 by data exchange through the Favorite Browser.

This result supports the accuracy and robustness of the funRNA gene identification pipeline. According to the prediction results, the average numbers of genes encoding 
Argonaute and Dicer were significantly higher in Metazoa and Viridiplantae than in Fungi (t-test: $P \leq 2.53 \mathrm{e}^{-6}$ for Argonaute and $P \leq 7.38 \mathrm{e}^{-5}$ for Dicer). In the case of RdRP, the Viridiplantae kingdom presented the largest average number of genes (5.09), followed by Fungi (2.04) and Metazoa (1.27) (t-test: $\left.P \leq 2.45 \mathrm{e}^{-4}\right)$. No genes encoding Argonaute, Dicer, or RdRP were detected in 1,059 and 51 proteomes of bacteria and archaea, respectively. However, two archaeal species (Methanocaldococcus jannaschii DSM 2661 and Pyrococcus furiosus DSM 3638) and one bacterial species (Aquifex aeolicus VF5) were predicted to have an Argonaute-encoding gene. In fact, PfAgo, an Argonaute found in P. furiosus DSM 3638, has been structurally characterized by X-ray crystallography [17], and was correctly captured by the pipeline.

In fungi, species belonging to the subphylum Agaricomycotina showed a higher number of genes in all gene families than any other fungal subphylum, with 5.68 Argonaute, 2.46 Dicer, and 6.93 RdRP genes on average (Figure 2 andTable 1). Putative genes were not predicted in the species belonging to the subphylum Ustilaginomycotina, in agreement with previous reports $[19,20]$. In the phylum Ascomycota, the majority of genes were found in species belonging to the subphylum Pezizomycotina. Species

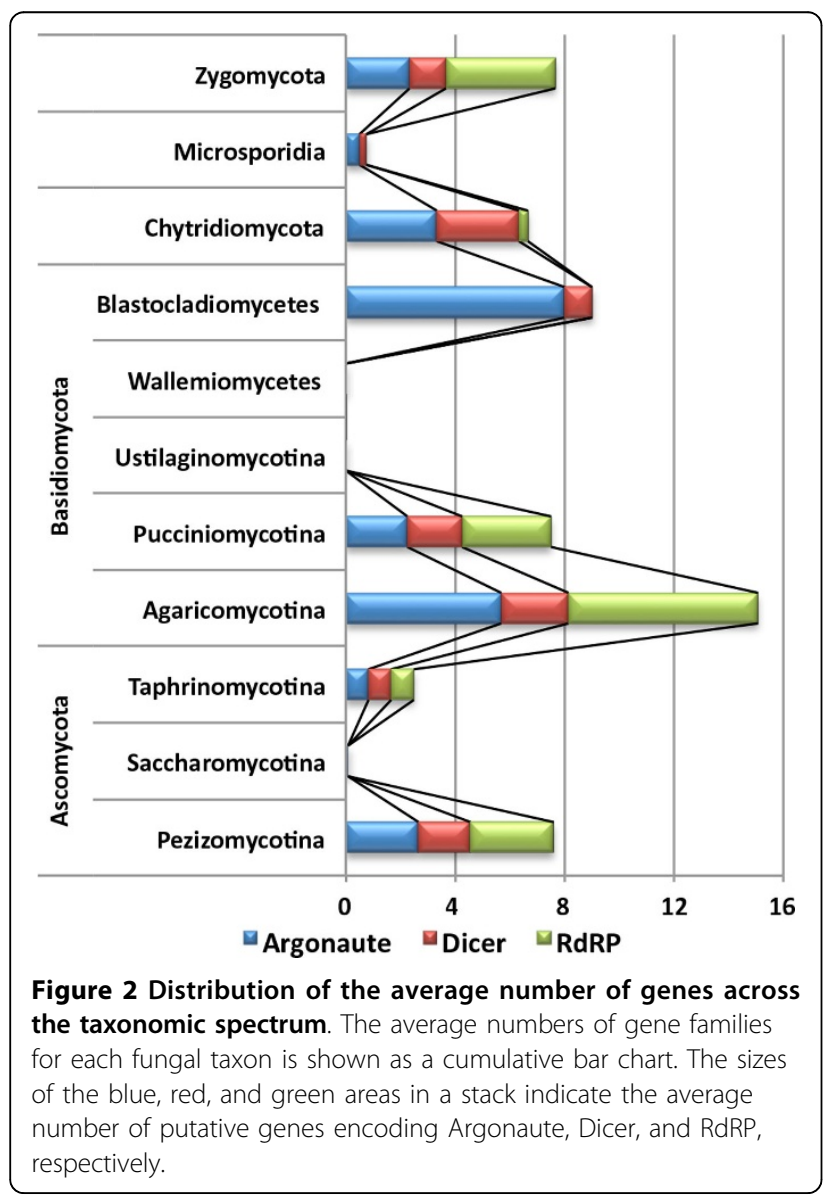

belonging to the subphylum Saccharomycotina, including five genomes of Candida spp., had only a few genes encoding Argonaute and no genes for Dicer and RdRP. Although a recent paper reported the presence of RNAi in Saccharomyces castellii and C. albicans, these species use non-canonical Dicers to generate small interfering RNAs [21]. Meanwhile, Schizosaccharomyces spp., belong to the subphylum Taphrinomycotina, had one gene for each of three gene families (Additional file 1).

In Plasmodium spp., no genes were predicted to encode the key players of RNAi. This is in accordance with previous studies performed with protozoan parasites including Trypanosoma cruzi, Leishmania major, L. donovani, and Plasmodium spp. [22,23]. The only exception is the RNA-mediated gene silencing in P. falciparum [24,25]. Accordingly, it was speculated that Plasmodium spp. might possess a non-canonical RNAi pathway that is yet to be characterized [26].

While the three genes were frequently predicted in two fungal subphyla, Pezizomycotina and Agaricomycotina, the number of genes in the latter subphylum varied more than that in the former. The greatest variance was found in the genes encoding RdRP. The number of RdRP genes in the Pezizomycotina species ranged from two to five, with a standard deviation of 0.64; for Agaricomycotina the range was zero to 14 , with a standard deviation of 3.63 . Interestingly, no putative genes encoding RdRP were found in two metazoan phyla, Arthropoda (except for in Ixodes scapularis, the blacklegged tick) and Chordata, to which fruit flies and humans belong, respectively. Considering the possibility that virus-encoded RdRPs may play a role in RNAi-like antiviral activity in plants [27], we speculated that the same could be happening in Drosophila spp. and mammals. This was supported by the fact that mouse oocytes with a horizontally transferred RdRP from a virus exhibited RNAi [28]. By contrast, worm species belonging to the phylum Nematoda had multiple genes with copy numbers ranging from three to eight. Meanwhile, in the Viridiplantae kingdom, a clear distinction in the number of genes was found between the Chlorophyta and Streptophyta phyla. Streptophyta species had higher average numbers of the three genes (13.55 for Argonaute, 4.86 for Dicer, and 7.27 for RdRP) than Chlorophyta (0.90, 0.40, and 0.30 , respectively) (t-test: $P=1.60 \mathrm{e}^{-10}, 2.13 \mathrm{e}^{-08}$, and $1.69 \mathrm{e}^{-10}$, respectively). In Chlamydomonas reinhardtii, which belongs to the Chlorophyta, it was presumed that the absence of an RdRP gene is a reflection of its minimalistic genomic nature, and that it thus only exhibits the essential RNAi phenomenon [29]. It was also speculated that $C$. reinhardtii only recognizes dsRNA to trigger RNAi, since the transformation of single-stranded RNA favors non-homologous recombination [30]. Notably, Lotus japonicus was predicted to have no genes encoding Dicer. In fact, two genes encoding Dicer-like proteins were 
Table 1 Summary of the average number of genes per genome across the taxonomic spectrum.

\begin{tabular}{|c|c|c|c|c|c|}
\hline Kingdom & Phylum & Subphylum & Argonaute $^{*}$ & Dicer $^{*}$ & RdRP* $^{*}$ \\
\hline Chromalveolata & & & 0 & 0 & $0.15(0-2)$ \\
\hline Chromista & & & $2.75(0-6)$ & $0.25(0-1)$ & $0.88(0-3)$ \\
\hline \multirow[t]{11}{*}{ Fungi } & Ascomycota & Pezizomycotina & $2.66(1-7)$ & $1.9(0-3)$ & $3.04(2-5)$ \\
\hline & & Saccharomycotina & $0.08(0-1)$ & 0 & 0 \\
\hline & & Taphrinomycotina & $0.83(0-1)$ & $0.83(0-1)$ & $0.83(0-1)$ \\
\hline & Basidiomycota & Agaricomycotina & $5.68(0-10)$ & $2.46(0-4)$ & $6.93(0-14)$ \\
\hline & & Pucciniomycotina & $2.25(2-3)$ & $2(0-5)$ & $3.25(1-5)$ \\
\hline & & Ustilaginomycotina & 0 & 0 & 0 \\
\hline & & Wallemiomycetes ${ }^{* *}$ & 0 & 0 & 0 \\
\hline & & Blastocladiomycetes $^{* *}$ & 8 & 1 & 0 \\
\hline & Chytridiomycota & N/D & $3.33(2-6)$ & $3.00(3)$ & $0.33(0-1)$ \\
\hline & Microsporidia & N/D & $0.50(0-1)$ & $0.25(0-1)$ & 0 \\
\hline & Zygomycota & Mucoromycotina & $2.33(2-3)$ & $1.33(1-2)$ & $4.00(3-5)$ \\
\hline Metazoa & & & $9.88(1-49)$ & $2.24(0-7)$ & $1.27(0-9)$ \\
\hline \multirow[t]{2}{*}{ Viridiplantae } & Chlorophyta & & $0.90(0-2)$ & $0.40(0-2)$ & $0.30(0-1)$ \\
\hline & Streptophyta & & $13.55(6-25)$ & $4.86(0-11)$ & $7.27(3-14)$ \\
\hline $\begin{array}{l}\text { Other } \\
\text { eukaryotes }\end{array}$ & & & $1.76(0-14)$ & $0.29(0-3)$ & $0.76(0-4)$ \\
\hline
\end{tabular}

* Range of number of genes is shown in parentheses.

** Class name is shown if no subphylum name is assigned.

predicted to have RNase III and dsRNA-binding domains. They had only one RNase III domain, while canonical Dicers are known to contain two separate RNase III domains. Because experimental evidence shows that functional RNAi is present in L. japonicus [31,32], the two genes may encode real Dicers with a simpler domain structure. In addition, monocot plants tend to have more Argonaute genes than dicots. Actually, orthologs of Dicerlike genes in Oryza sativa were found in other monocot plants, such as Zea mays and Saccharum officinarum, but not in Arabidopsis thaliana, supporting gene duplication after monocot and dicot divergence [33].

\section{Evolutionary history of gene duplication/loss and active sites residues in Argonautes}

The identification results showed that Argonaute-encoding genes were found in many fungal species in the subphyla Pezizomycotina and Agaricomycotina, as well as in plants and animals (Additional file 1). In order to elucidate evolutionary footprints, reconciliation analysis was performed with a species tree and an Argonaute gene tree. A total of 34 species predicted to have Argonaute-encoding genes were subjected to the analysis, which covered species belonging to multiple kingdoms, including Fungi, Viridiplantae, Metazoa, Bacteria, and Archaea (Table 2). Massive gene duplication events were found in the animal and plant species. In fungi, however, duplications were found only in basidiomycetes and two ascomycetes.

Catalytic amino acid residues for Argonaute slicer activity were characterized in previous studies $[17,34,35]$.
Argonaute sequences found in Homo sapiens, A. thaliana, $D$. melanogaster, S. pombe, and C. elegans were analyzed for catalytic residues, or the DDH motif [36]. In addition, functional variants of the DDH motif were experimentally identified, giving a relaxed motif definition, DD[HDEK] $[34,37]$. Archaea, as the most divergent species evolutionarily, have a totally different composition of active site amino acids compared to other species (Figure 3). The DD [HDEK] motif was found in Argonaute sequences from a bacterium (Aquifex aeolicus), fungi, plants, and animals. In fungi, the first two residues were well conserved. The third residue was variable, but was predominantly aspartic acid (Figure 3 and Additional file 2). Dicers in C. elegans, $H$. sapiens, and $O$. sativa have been through more specieslevel duplications, which possibly resulted in greater residue variation in the catalytic motif. The DDH triad was the most frequent motif in Argonautes of animals and plants; most fungus Argonautes had a DD[DEK] motif. "The Others", sequences showing aligned residues other than DD[HDEK], were much more common in animal species, especially $C$. elegans, possibly due to gene diversification resulting from a number of species-level duplication events.

\section{Differential distribution of accessory domains in putative \\ Dicers}

In plants, the copy numbers of genes encoding Dicer-like proteins increased during their evolution, with diversification occurring by duplication and transposition [38]. In fact, our data also show diversification of Dicer-like genes 
Table $\mathbf{2}$ List of species selected for sequence analysis of RdRPs and reconciliation analysis of Argonautes.

\begin{tabular}{|c|c|c|c|}
\hline Species name & Taxonomy & Argonaute gene & RdRP gene \\
\hline Methanocaldococcus jannaschii DSM 2661 & Archaea $>$ Euryarchaeota $>$ N/D & 1 & 0 \\
\hline Pyrococcus furiosus DSM 3638 & Archaea $>$ Crenarchaeota $>$ N/D & 1 & 0 \\
\hline Aquifex aeolicus VF5 & Bacteria $>$ Aquificae $>N / D$ & 1 & 0 \\
\hline Aspergillus fumigatus Af293 & Fungi>Ascomycota $>$ Pezizomycotina & 2 & 2 \\
\hline Aspergillus nidulans & Fungi>Ascomycota $>$ Pezizomycotina & 1 & 2 \\
\hline Botrytis cinerea & Fungi>Ascomycota>Pezizomycotina & 2 & 2 \\
\hline Coccidioides immitis RS & Fungi>Ascomycota $>$ Pezizomycotina & 3 & 3 \\
\hline Colletotrichum graminicola M1.001 & Fungi>Ascomycota $>$ Pezizomycotina & 2 & 3 \\
\hline Fusarium graminearum & Fungi>Ascomycota $>$ Pezizomycotina & 2 & 5 \\
\hline Fusarium oxysporum & Fungi>Ascomycota $>$ Pezizomycotina & 5 & 5 \\
\hline Histoplasma capsulatum H88 & Fungi $>$ Ascomycota $>$ Pezizomycotina & 2 & 3 \\
\hline Magnaporthe oryzae 70-15 & Fungi>Ascomycota $>$ Pezizomycotina & 3 & 3 \\
\hline Mycosphaerella graminicola & Fungi>Ascomycota $>$ Pezizomycotina & 4 & 2 \\
\hline Neurospora crassa & Fungi $>$ Ascomycota $>$ Pezizomycotina & 2 & 3 \\
\hline Podospora anserina & Fungi>Ascomycota $>$ Pezizomycotina & 2 & 4 \\
\hline Candida albicans & Fungi $>$ Ascomycota $>$ Saccharomycotina & 1 & 0 \\
\hline Schizosaccharomyces pombe & Fungi>Ascomycota>Taphrinomycotina & 1 & 1 \\
\hline Heterobasidion irregulare TC 32-1 & Fungi>Basidiomycota>Agaricomycotina & 7 & 7 \\
\hline Laccaria bicolor & Fungi>Basidiomycota $>$ Agaricomycotina & 6 & 6 \\
\hline Phanerochaete chrysosporium & Fungi>Basidiomycota $>$ Agaricomycotina & 6 & 8 \\
\hline Serpula lacrymans & Fungi>Basidiomycota $>$ Agaricomycotina & 6 & 6 \\
\hline Cryptococcus neoformans var. grubii H99 & Fungi $>$ Basidiomycota $>$ Agaricomycotina & 1 & 1 \\
\hline Melampsora laricis-populina & Fungi>Basidiomycota $>$ Pucciniomycotina & 2 & 5 \\
\hline Puccinia graminis & Fungi $>$ Basidiomycota $>$ Pucciniomycotina & 2 & 5 \\
\hline Allomyces macrogynus & Fungi>Blastocladiomycota $>N / D$ & 8 & 0 \\
\hline Batrachochytrium dendrobatidis JAM81 & Fungi $>$ Chytridiomycota>N/D & 2 & 0 \\
\hline Phycomyces blakesleeanus & Fungi>Zygomycota $>$ Mucoromycotina & 2 & 4 \\
\hline Rhizopus oryzae & Fungi>Zygomycota $>$ Mucoromycotina & 2 & 5 \\
\hline Phytophthora infestans & Chromista>Oomycota>Oomycotina & 5 & 1 \\
\hline Arabidopsis thaliana & Viridiplantae $>$ Streptophyta>N/D & 14 & 6 \\
\hline Oryza sativa & Viridiplantae $>$ Streptophyta $>$ N/D & 25 & 6 \\
\hline Drosophila melanogaster & Metazoa>Arthropoda>N/D & 12 & 0 \\
\hline Caenorhabditis elegans & Metazoa $>$ Nematoda $>$ N/D & 31 & 4 \\
\hline Homo sapiens & Metazoa $>$ Chordata $>$ Craniata & 17 & 0 \\
\hline
\end{tabular}

in plants, including Glycine max, Z. mays, O. sativa, Brachypodium distachyon, and $A$. thaliana. Diversification of Dicer often presented as alternatively spliced genes that produce multiple products (Additional file 3). Interestingly, this was true for species belonging to the phylum Streptophyta, but not for Chlorophyta. Only 3 of 10 Chlorophyta species were predicted to have one or two Dicerencoding genes, while the other seven were not predicted to have any Dicer genes (Additional file 1). This confirms the previous finding that diversification of Dicers in plants occurred before the divergence of monocot and dicot plants, but after the divergence of green algae and plants [38]. Even though a few fungal genomes provided alternatively spliced transcript information, fungal Dicers showed no evidence of diversification by alternative splicing. In $N$. crassa, Magnaporthe oryzae 70-15, and Cryptococcus neoformans var. grubii $\mathrm{H} 99$, for example, no alternatively spliced form was found for the putative Dicer-encoding genes.

Besides the essential RNase III and dsRNA-binding domains (Table 3), the other 50 additional domains were not universally present in the Dicers in plants, animals, and fungi (Additional file 4). On average, each had 5.28 additional domains other than the essential ones. The genes of the Viridiplantae species showed the highest number of additional domains (6.41), followed by those of Metazoa (5.12) and Fungi (4.87). The top six most frequent additional domains were IPR001650 (Helicase, Cterminal), IPR014001 (DEAD-like helicase), IPR011545 (DNA/RNA helicase, DEAD/DEAH box type, $\mathrm{N}$-terminal), IPR003100 (Argonaute/Dicer protein, PAZ), IPR006935 (UvrABC complex, subunit B), and IPR014720 (Double- 


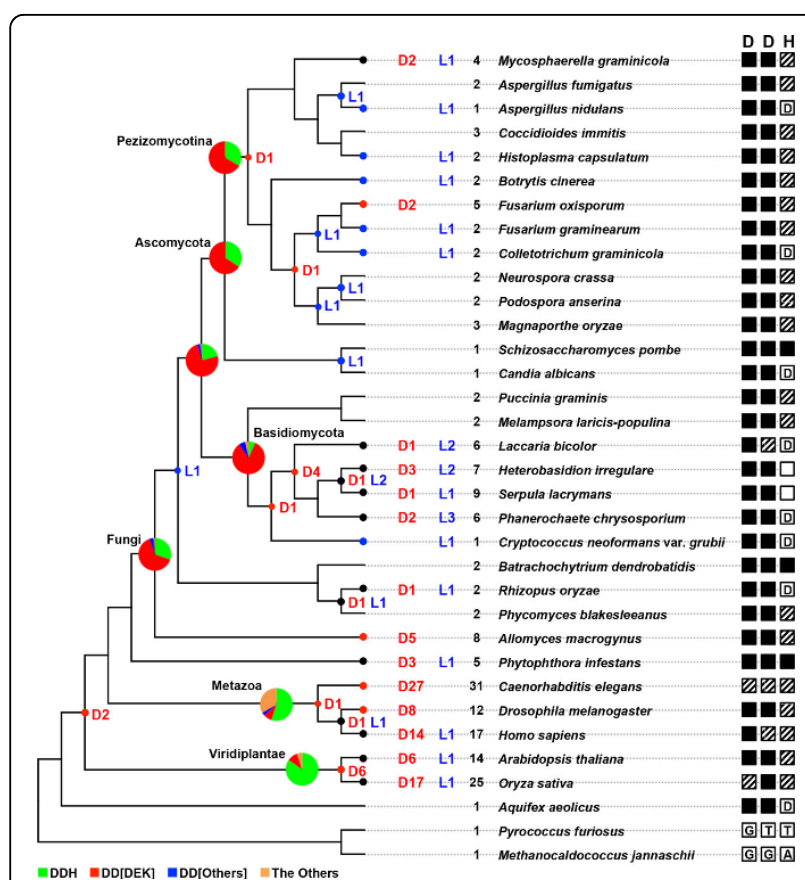

Figure 3 Duplication and loss of Argonaute genes and variation of the catalytic motif. Gene duplication and loss events were estimated by reconciliation analysis. Red and blues dots are shown at internal nodes if duplication and loss were predicted, respectively. Black dots indicate nodes where both duplication and loss were discovered. Numbers of species-level duplication and loss events, and the number of putative genes encoding Argonaute, are shown between the tree and species name. In the rightmost column, amino acid variation of the DDH motif is shown with symbols: i) filled squares indicate that all the genes in the corresponding species had the conserved reference residue; ii) shaded squares indicate existence of the conserved residue and variants; iii) empty squares indicate variants without the conserved residue; and iv) single-letter amino acid codes indicate conserved residues, but not the reference amino acid. For the complete list of partial alignments near each amino acid, see Additional file 2. Pie charts shown in the internal nodes display the distribution of DDH motif variants for each taxon specified. The proportion of genes containing the conserved DDH motif is shown in green; $\mathrm{H}$ substituted by $\mathrm{D}, \mathrm{E}$, or $\mathrm{K}$ is shown in red; $\mathrm{H}$ substituted by another amino acid is shown in blue; and other variants are shown in orange. stranded RNA-binding-like) (Additional file 4). Among the 50 domains, the distribution of three dsRNA-binding domains (InterPro accession numbers IPR001159, IPR005034, and IPR014720) varied across the taxonomic spectrum. All fungal Dicers had an IPR005034 domain, but those in Dictyostelium spp., animal, and plant species often lacked the domain (Additional file 4). The IPR001159 domain was present in 20.74\% (28 of 135) of the predicted sequences from species belonging to the subphylum Pezizomycotina. By contrast, there was no Dicer containing the IPR001159 domain in species belonging to the subphylum Agaricomycotina. Meanwhile, Metazoa and Viridiplantae species had one or both of the IPR001159 and IPR005034 domains. The IPR014720 domain was only detected in genes from two fungi (Trichoderma atroviride and Mucor circinelloides), while $46.31 \%$ (94 of 203) of metazoan and plant Dicers were predicted to have the domain. Interestingly, the PAZ domain (IPR003100), named after the proteins Piwi, Argonaute, and Zwille, was rarely found in fungi (nine out of 232 proteomes), but more often in metazoan and plant species (51/92 and 99/111, respectively). Although the threedimensional structure of the PAZ domain has been resolved [39-41], its function is not clear, although it has been speculated that it may mediate the formation of complexes between proteins of the Piwi and Dicer families by heterodimerization [42]. Future research may focus on the functionality of Dicers without the PAZ domain to demonstrate the essentiality of the domain in fungi.

Differences in domain composition were also reflected in a phylogenetic tree that was constructed using the 442 Dicer sequences (Additional file 3). It is noteworthy that the tree was taxonomically divided into four clades: two Metazoa-dominant clades, one plant-dominant clade, and one fungus-dominant clade. In plant species, isoform products were grouped together closely, supporting the diversification reported previously [38]. Interestingly, the putative Dicers from metazoan species formed two distinct clades, one containing minimal domains and the other containing multiple additional domains (Additional files 3

Table 3 Domain profile definitions used in funRNA

\begin{tabular}{lllll}
\hline Gene family & InterPro accession number & Domain description & Number of genes $^{*}$ & Number of genomes $^{*}$ \\
\hline Argonaute & IPR003100 & Argonaute/Dicer protein, PAZ & 1,163 & 209 \\
& & & $(396)$ & $(122)$ \\
& IPR003165 & Stem cell self-renewal protein Piwi & 180 \\
\hline Dicer & IPR000999 & Ribonuclease II & 442 & $(111)$ \\
& IPR001159* & & & \\
& IPR005034* & Double-stranded RNA-binding & & \\
& IPR007855 & Dicer double-stranded RNA-binding fold & & 157 \\
\hline RdRP & RNA-dependent RNA polymerase, eukaryotic type & 678 & $(441)$ & $(111)$ \\
\hline
\end{tabular}

\footnotetext{
* Numbers of fungal genes/genomes are shown in parentheses.

** Genes containing one of two double-stranded RNA-binding domains were predicted to be Dicer-encoding genes.
} 
and 4). The two Metazoa-dominant clades suggest that minimal Dicer could be the ancestral form, which acquired additional domains during the evolution of individual organisms. Most of the fungal and plant Dicers possessed multiple additional domains.

\section{Structural conservation analysis of residues in catalytic regions of RdRPs in fungi}

In $N$. crassa, a $2.3-\AA$-resolution crystal structure of an RdRP (QDE-1) was characterized [43]. QDE-1 was structurally aligned with the protein sequences of bacterial and yeast polymerases. Structurally conserved catalytic motifs, including double-psi $\beta$-barrels (DPBB1 and 2), with multiple invariant residues were found. To test the conservation of such amino acid residues, a multiple sequence alignment was performed with 84 putative RdRP sequences from selected fungal proteomes (Table 2), including QDE1 . When counting the residues with $70 \%$ or higher conservation, 13 and 22 residues were found to be conserved in DPBB1 and DPBB2 based on the positions in QDE-1, respectively. Some were also reported to be conserved in a previous study. For example, three aspartic acid residues (D) located at positions 1,007, 1,009, and 1,011 in QDE-1 were conserved in $84.52 \%$ of the sequences analyzed. The high conservation of these three aspartic acid residues reflects their importance in binding $\mathrm{Mg}^{2+}$ as a cofactor. Double glycine (G at positions 1,005 and 1,006 in QDE-1) was found in $83.33 \%$ of analyzed sequences, although not in bacterial and yeast polymerases [43] (Additional file 5).

\section{Utility}

\section{Web utility}

To provide a user-friendly interface, funRNA adopted the Data-driven User Interface powered by the Comparative Fungal Genomics Platform 2.0 (CFGP 2.0; http://cfgp.snu. ac.kr/) [44]. The genes identified by the pipeline can be browsed by species or gene family. The reference sequences used in pipeline construction are also available. The detail information page for each gene shows the gene structure, sequence information, domain structure, GO terms, information on similarity to the reference sequences, and results from seven additional bioinformatics programs. The statistics page of "Species Browser" provides a kingdom-/subphylum-level summary, giving a glimpse into the macro-taxonomic distribution. funRNA also provides analysis functions, including: i) sequence similarity searches (BLAST [45] and BLASTMatrix [44]); ii) multiple sequence alignment (ClustalW [46]) with fulllength or domain regions; and iii) protein domain analysis and download functions (Figure 4).

\section{Extended analyses through sister web-based systems} funRNA supports "Favorite Browser", a personalized virtual storage and analysis hub that was originally developed in CFGP 2.0 [44]. Sequences archived in funRNA have the same identifiers as those used in CFGP 2.0, enabling flexible data exchange with CFGP 2.0, as well as with sister databases [47-50]. In Favorite Browser in CFGP 2.0, 27 bioinformatics tools are currently available, providing broader analysis options for the sequences collected in funRNA. For example, a sequence collection created in funRNA could be analyzed in Favorite Browser in CFGP 2.0 to find conserved motifs by using the MEME program [51].

\section{Conclusions}

funRNA is a web-based workbench that provides an analysis environment for the key components of RNAi. funRNA provides: i) a putative gene archive from 1,440 proteomes over a wide taxonomic range; ii) graphs and summary tables for an overview of the target gene families; iii) detailed information about the predicted genes; iv) job submission forms for bioinformatics tools; and v) Favorite synchronization with CFGP 2.0 and sister databases to offer further analysis. In addition, diverse comparative analyses can be conducted, such as the analyses shown in this paper. In summary, funRNA is a useful resource for comparative and evolutionary genomics analyses of Argonaute, Dicer, and RdRP genes.

\section{Methods}

\section{Establishment of protein domain profiles}

In order to determine protein domain profiles for the genes encoding Argonaute, Dicer, and RdRP, annotated

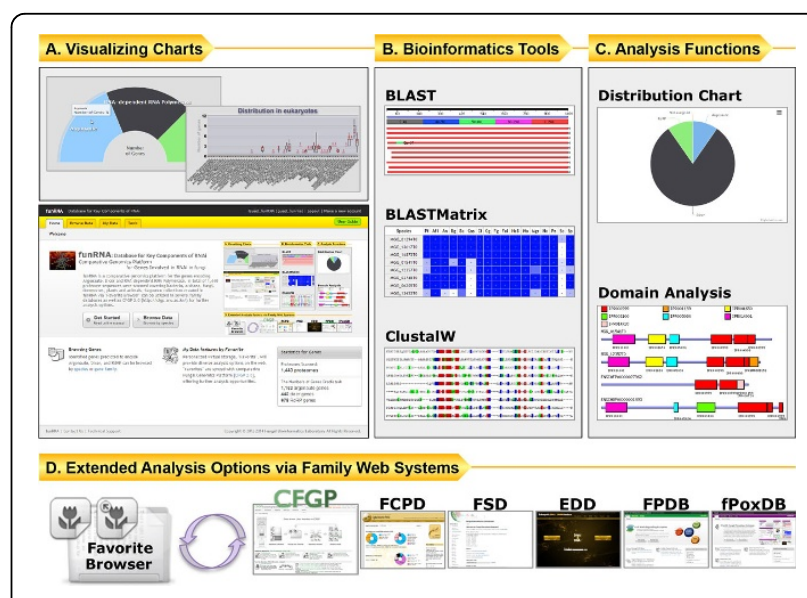

Figure 4 Functionalities of the funRNA website. A) Web interface of funRNA displays graphical charts for better recognition of the distribution of genes. B) Tools including similarity search tools (BLAST and BLASTMatrix) and a multiple sequence alignment tool (ClustalW) are provided via the Favorite Browser. C) Protein domain analysis can be conducted with the sequences collected in

Favorites. D) Users' sequence collections can be further analyzed by the tools available in CFGP 2.0 and other sister databases. 
protein sequences were retrieved from the UniProtKB/ SwissProt database [18]. In total, 50, 44, and 13 sequences belonging to the respective gene families were subjected to domain profiling using InterPro scan (version 4.8) [52]. For each gene family, commonly shared domains were determined and used for prediction of putative genes (Table 3). The domain profiles acquired from the reference sequences were consistent with previous findings [53,54]. According to previous studies, Argonautes contain PIWI and PAZ domains [36], and fungal Dicer and Dicerlike proteins have RNase III and double-stranded RNA binding domains [53]. For Dicer identification, sequences with only one RNase III domain were discarded from the final prediction to improve the results. All sequences used to construct the pipeline are available from "Reference Sequences" under the "Browse Data" menu.

\section{Preparation of proteome sequences to be searched}

To identify genes involved in small RNA processing, 1,440 proteomes were scanned with the protein domain profiles (Additional file 1). The target proteomes included 221 fungal/Oomycete genomes, as well as 1,060 bacterial, 53 archaeal, 32 plant, and 41 metazoan proteomes to investigate evolutionary traces in other kingdoms (Additional file 1). All the proteome sequences were obtained from the standardized genome data warehouse in CFGP $2.0 \mathrm{http} / / /$ cfgp.snu.ac.kr/ [44].

\section{Evaluation of the pipeline}

To evaluate the gene identification pipeline, sequences annotated as Argonaute, Dicer, and RdRP were obtained from UniProtKB/TrEMBL [18]. They did not include sequences used in domain profile determination. The test set consisted of 425 Argonaute, 209 Dicer, and 227 RdRP protein sequences. The sequences were scanned using InterPro scan [52], and searched with the funRNA domain profiles to assess the accuracy. Considering the average length of the sequences used in defining the domain profiles (942 aa for Argonaute, 1,575 aa for Dicer, and 1,081 aa for RdRP), sequences shorter than 500 aa were discarded from the test set.

\section{Assessment of gene duplication and loss}

A species phylogeny was created by using CVTree (version 4.2.1; source code distribution) [55]. Whole proteome sequences of the target species were used as the input, and $\mathrm{K}$-tuple length was set to seven, which is known to be optimal for fungal phylogeny construction $[56,57]$. The output distance matrix was converted into a neighborjoining tree by using neighbor in the PHYLIP package (version 3.69) [58]. To build gene trees, multiple sequence alignment was performed by using MUSCLE in MEGA6 [59]. Subsequently, a phylogenetic tree was constructed with the Minimum Evolution algorithm by using MEGA6 [59]. To investigate gene duplication and loss events, reconciliation analysis was conducted by using Notung software (version 2.6) [60] with the species and gene trees. A total of 34 genomes were subjected to the reconciliation analysis, comprising 25 fungi, one Oomycete, one bacterium, two archaea, two plants, and three animals. Non-fungal species were also included to better understand the evolutionary history (Table 2).

\section{Multiple sequence alignment, phylogenetic tree} construction, and visualization of conserved sequences

Full-length protein sequences of the 442 predicted Dicers were aligned using ClustalW [46]. A phylogenetic tree was constructed using MEGA6 [59] by the Minimum Evolution method with 10,000 bootstrap replicates.

In order to detect conservation of amino acid residues, 84 RdRP sequences were aligned using M-Coffee [61]. One putative RdRP-encoding gene, FOXG_00217 in Fusarium oxysporum, was excluded from the analysis because of its very short domain region (68 aa). Sequence logos were created by using WebLogo [62].

\section{Availability of supporting data}

All data described in this paper can be freely accessed at the funRNA website http://funrna.riceblast.snu.ac.kr/ using the latest versions of Google Chrome, Mozilla Firefox, Microsoft Internet Explorer ( 9 or higher), and Apple Safari. The data sets supporting the results of this article are included in the article and its additional files.

\section{Additional material}

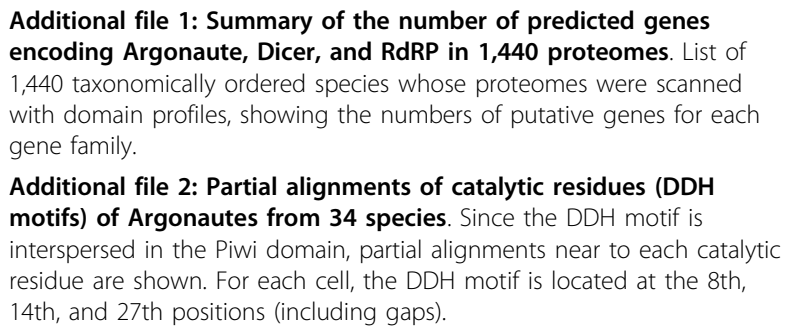

Additional file 2: Partial alignments of catalytic residues (DDH motifs) of Argonautes from 34 species. Since the DDH motif is interspersed in the Piwi domain, partial alignments near to each catalytic residue are shown. For each cell, the DDH motif is located at the 8th, 14th, and 27th positions (including gaps)

Additional file 3: Phylogenetic tree constructed with 442 Dicer sequences predicted from 180 proteomes. A total of 442 full-length Dicer sequences were used to construct a phylogenetic tree. The tree could be divided into four major clades, two of which were predominant for animals, and one each for plants and fungi. A Metazoa-dominant clade with minimal domains is shown in pink; the other metazoan clade is shown in red. The Viridiplantae-dominant clade is shown in green and the fungal clade in blue.

Additional file 4: Distribution of auxiliary domains found in $\mathbf{4 4 2}$ Dicer sequences. Presence and absence table of additional domains found in Dicer sequences. " $O$ " indicates the presence of the corresponding domain and " $X$ " absence.

Additional file 5: Sequence logos based on multiple sequence alignment of $\mathbf{8 4}$ putative RdRP sequences. Sequence logos for double-psi $\beta$-barrels (DPBB1 and 2) and the flap sub-domain based on a 
multiple sequence alignment of 84 sequences including an RdRP from $\mathrm{N}$. crassa (QDE-1). Amino acid residues with $70 \%$ or more conservation are highlighted in red.

\section{Competing interests}

The authors declare that they have no competing interests.

\section{Authors' contributions}

$J C$ and YHL designed the project. JC developed the pipeline and database. $\mathrm{JC}$ and $\mathrm{HS}$ developed the web interfaces. JC, KTK, and JJ conducted the data analysis. JC, KTK, JJ, JW, HS, FOA, and YHL wrote the manuscript. All the authors read and approved the final manuscript.

\section{Acknowledgements}

This work was supported by National Research Foundation of Korea grants funded by the Korean government (2008-0061897 and 2013-003196), and by the Cooperative Research Program for Agriculture Science \& Technology Development (project no. PJ00821201), Rural Development Administration, Republic of Korea. JC, KTK, JJ, and HS are grateful for a graduate fellowship through the Brain Korea 21 Plus Program. This work was also supported by the Finland Distinguished Professor Program (FiDiPro) of the Academy of Finland (FiDiPro \# 138116)

This article has been published as part of BMC Genomics Volume 15 Supplement 9, 2014: Thirteenth International Conference on Bioinformatics (InCoB2014): Computational Biology. The full contents of the supplement are available online at http://www.biomedcentral.com/bmcgenomics/ supplements/15/S9.

\section{Authors' details}

'Center for Fungal Pathogenesis, Seoul National University, Seoul 151-921, Korea. ${ }^{2}$ Fungal Bioinformatics Laboratory, Department of Agricultural Biotechnology, Seoul National University, Seoul 151-921, Korea. ${ }^{3}$ Department of Forest Sciences, University of Helsinki, 00014 Helsinki, Finland. ${ }^{4}$ Center for Fungal Genetic Resources, Plant Genomics and Breeding Institute, Research Institute for Agriculture and Life Sciences, Seoul National University, Seoul 151-921, Korea.

\section{Published: 8 December 2014}

\section{References}

1. Fire A, Xu S, Montgomery MK, Kostas SA, Driver SE, Mello CC: Potent and specific genetic interference by double-stranded RNA in Caenorhabditis elegans. Nature 1998, 391(6669):806-811.

2. Carmell MA, Xuan ZY, Zhang MQ, Hannon GJ: The Argonaute family: tentacles that reach into RNAi, developmental control, stem cell maintenance, and tumorigenesis. Genes Dev 2002, 16(21):2733-2742.

3. Ding SW, Voinnet O: Antiviral immunity directed by small RNAs. Cell 2007, 130(3):413-426.

4. Ghildiyal M, Zamore PD: Small silencing RNAs: an expanding universe. Nat Rev Genet 2009, 10(2):94-108.

5. Li LD, Chang SS, Liu Y: RNA interference pathways in filamentous fungi. Cell Mol Life Sci 2010, 67(22):3849-3863.

6. Romano N, Macino G: Quelling: transient inactivation of gene expression in Neurospora crassa by transformation with homologous sequences. Mol Microbiol 1992, 6(22):3343-3353.

7. Cogoni C, Irelan JT, Schumacher M, Schmidhauser TJ, Selker EU, Macino G: Transgene silencing of the al-1 gene in vegetative cells of Neurospora is mediated by a cytoplasmic effector and does not depend on DNA-DNA interactions or DNA methylation. EMBO J 1996, 15(12):3153-3163.

8. Carattoli A, Cogoni C, Morelli G, Macino G: Molecular characterization of upstream regulatory sequences controlling the photoinduced expression of the albino-3 gene of Neurospora crassa. Mol Microbiol 1994, 13(5):787-795.

9. Cogoni C, Macino G: Isolation of quelling-defective (qde) mutants impaired in posttranscriptional transgene-induced gene silencing in Neurospora crassa. Proc Natl Acad Sci USA 1997, 94(19):10233-10238.
10. Cogoni C, Macino G: Gene silencing in Neurospora crassa requires a protein homologous to RNA-dependent RNA polymerase. Nature 1999, 399(6732):166-169.

11. Cogoni C, Macino G: Posttranscriptional gene silencing in Neurospora by a RecQ DNA helicase. Science 1999, 286(5448):2342-2344.

12. Catalanotto C, Pallotta M, ReFalo P, Sachs MS, Vayssie L, Macino G, Cogoni $C$ : Redundancy of the two Dicer genes in transgene-induced posttranscriptional gene silencing in Neurospora crassa. Mol Cell Biol 2004, 24(6):2536-2545.

13. Lee DW, Pratt RJ, McLaughlin M, Aramayo R: An argonaute-like protein is required for meiotic silencing. Genetics 2003, 164(2):821-828.

14. Shiu PK, Raju NB, Zickler D, Metzenberg RL: Meiotic silencing by unpaired DNA. Cell 2001, 107(7):905-916.

15. Volpe TA, Kidner C, Hall IM, Teng G, Grewal SI, Martienssen RA: Regulation of heterochromatic silencing and histone $\mathrm{H} 3$ lysine- 9 methylation by RNAi. Science 2002, 297(5588):1833-1837.

16. Nakayashiki H, Kadotani N, Mayama S: Evolution and diversification of RNA silencing proteins in fungi. J Mol Evol 2006, 63(1):127-135.

17. Song JJ, Smith SK, Hannon GJ, Joshua-Tor L: Crystal structure of Argonaute and its implications for RISC slicer activity. Science 2004, 305(5689):1434-1437.

18. Apweiler R, Bateman A, Martin MJ, O'Donovan C, Magrane M, AlamFaruque Y, Alpi E, Antunes R, Arganiska J, Casanova EB, Bely B, Bingley M, Bonilla C, Britto R, Bursteinas B, Chan WM, Chavali G, Cibrian-Uhalte E, Da Silva A, De Giorgi M, Fazzini F, Gane P, Castro LG, Garmiri P, Hatton-Ellis E, Hieta R, Huntley R, Legge D, Liu WD, Luo J, et al: Activities at the Universal Protein Resource (UniProt). Nucleic Acids Res 2014, 42(D1):D191-D198.

19. Laurie JD, Linning R, Bakkeren G: Hallmarks of RNA silencing are found in the smut fungus Ustilago hordei but not in its close relative Ustilago maydis. Curr Genet 2008, 53(1):49-58.

20. Laurie JD, Ali S, Linning R, Mannhaupt G, Wong P, Guldener U, Munsterkotter M, Moore R, Kahmann R, Bakkeren G, Schirawski J: Genome comparison of barley and maize smut fungi reveals targeted loss of RNA silencing components and species-specific presence of transposable elements. Plant Cell 2012, 24(5):1733-1745.

21. Drinnenberg $I A$, Weinberg $D E$, Xie $K T$, Mower JP, Wolfe KH, Fink GR, Bartel DP: RNAi in Budding Yeast. Science 2009, 326(5952):544-550.

22. Baum J, Papenfuss AT, Mair GR, Janse CJ, Vlachou D, Waters AP, Cowman AF, Crabb BS, de Koning-Ward TF: Molecular genetics and comparative genomics reveal RNAi is not functional in malaria parasites. Nucleic Acids Res 2009, 37(11):3788-3798.

23. Mueller AK, Hammerschmidt-Kamper C, Kaiser A: RNAi in Plasmodium. Curr Pharm Des 2014, 20(2):278-283.

24. McRobert L, McConkey GA: RNA interference (RNAi) inhibits growth of Plasmodium falciparum. Mol Biochem Parasitol 2002, 119(2):273-278.

25. Malhotra P, Dasaradhi PVN, Kumar A, Mohmmed A, Agrawal N, Bhatnagar RK, Chauhan VS: Double-stranded RNA-mediated gene silencing of cysteine proteases (falcipain-1 and-2) of Plasmodium falciparum. Mol Microbiol 2002, 45(5):1245-1254.

26. Schwentke A, Krepstakies M, Mueller AK, Hammerschmidt-Kamper C, Motaal BA, Bernhard T, Hauber J, Kaiser A: In vitro and in vivo silencing of plasmodial dhs and elf-5a genes in a putative, non-canonical RNAirelated pathway. BMC Microbiol 2012, 12:107.

27. Dalmay T, Hamilton A, Rudd S, Angell S, Baulcombe DC: An RNADependent RNA polymerase gene in Arabidopsis is required for posttranscriptional gene silencing mediated by a transgene but not by a virus. Cell 2000, 101(5):543-553.

28. Stein P, Svoboda P, Anger M, Schultz RM: RNAi: Mammalian oocytes do it without RNA-dependent RNA polymerase. RNA-Publ RNA Soc 2003, 9(2):187-192.

29. Schroda M: RNA silencing in Chlamydomonas: mechanisms and tools. Curr Genet 2006, 49(2):69-84.

30. Zorin B, Hegemann P, Sizova I: Nuclear-gene targeting by using singlestranded DNA avoids illegitimate DNA integration in Chlamydomonas reinhardtii. Eukaryot Cell 2005, 4(7):1264-1272.

31. Wang JC, Wang YM, Luo D: LjCYC genes constitute floral dorsoventral asymmetry in Lotus japonicus. J Integr Plant Biol 2010, 52(11):959-970.

32. Kumagai $H$, Kinoshita $E$, Ridge RW, Kouchi H: RNAi knock-down of ENOD40s leads to significant suppression of nodule formation in Lotus japonicus. Plant Cell Physiol 2006, 47(8):1102-1111. 
33. Kapoor M, Arora R, Lama T, Nijhawan A, Khurana JP, Tyagi AK, Kapoor S: Genome-wide identification, organization and phylogenetic analysis of Dicer-like, Argonaute and RNA-dependent RNA Polymerase gene families and their expression analysis during reproductive development and stress in rice. BMC Genomics 2008, 9:451.

34. Liu JD, Carmell MA, Rivas FV, Marsden CG, Thomson JM, Song JJ, Hammond SM, Joshua-Tor L, Hannon GJ: Argonaute2 is the catalytic engine of mammalian RNAi. Science 2004, 305(5689):1437-1441.

35. Rivas FV, Tolia NH, Song JJ, Aragon JP, Liu JD, Hannon GJ, Joshua-Tor L: Purified Argonaute2 and an siRNA form recombinant human RISC. Nat Struct Mol Biol 2005, 12(4):340-349.

36. Joshua-Tor L: The Argonautes. Cold Spring Harb Symp Quant Biol 2006, 71:67-72.

37. Meister G, Landthaler M, Patkaniowska A, Dorsett Y, Teng G, Tuschl T: Human Argonaute2 mediates RNA cleavage targeted by miRNAs and siRNAs. Mol Cell 2004, 15(2):185-197.

38. Margis R, Fusaro AF, Smith NA, Curtin SJ, Watson JM, Finnegan EJ, Waterhouse PM: The evolution and diversification of Dicers in plants. FEBS Lett 2006, 580(10):2442-2450.

39. Song JJ, Liu JD, Tolia NH, Schneiderman J, Smith SK, Martienssen RA, Hannon GJ, Joshua-Tor L: The crystal structure of the Argonaute2 PAZ domain reveals an RNA binding motif in RNAi effector complexes. Nat Struct Biol 2003, 10(12):1026-1032.

40. Yan KS, Yan S, Farooq A, Han A, Zeng L, Zhou MM: Structure and conserved RNA binding of the PAZ domain. Nature 2003, 426(6965):469-474.

41. Lingel A, Simon B, Izaurralde E, Sattler M: Structure and nucleic-acid binding of the Drosophila Argonaute 2 PAZ domain. Nature 2003, 426(6965):465-469.

42. Tahbaz N, Kolb FA, Zhang H, Jaronczyk K, Filipowicz W, Hobman TC: Characterization of the interactions between mammalian PAZ PIWI domain proteins and Dicer. EMBO Rep 2004, 5(2):189-194.

43. Salgado PS, Koivunen MRL, Makeyev EV, Bamford DH, Stuart DI, Grimes JM: The structure of an RNAi polymerase links RNA silencing and transcription. PLOS Biol 2006, 4(12):2274-2281.

44. Choi J, Cheong K, Jung K, Jeon J, Lee GW, Kang S, Kim S, Lee YW, Lee YH: CFGP 2.0: a versatile web-based platform for supporting comparative and evolutionary genomics of fungi and Oomycetes. Nucleic Acids Res 2013, 41(D1):D714-D719.

45. Johnson M, Zaretskaya I, Raytselis Y, Merezhuk Y, McGinnis S, Madden TL: NCBI BLAST: a better web interface. Nucleic Acids Res 2008, 36(W1):W5-W9.

46. Larkin MA, Blackshields G, Brown NP, Chenna R, McGettigan PA, McWilliam H, Valentin F, Wallace IM, Wilm A, Lopez R, Thompson JD, Gibson TJ, Higgins DG: Clustal W and Clustal X version 2.0. Bioinformatics 2007, 23(21):2947-2948

47. Choi J, Park J, Kim D, Jung K, Kang S, Lee YH: Fungal Secretome Database: Integrated platform for annotation of fungal secretomes. BMC Genomics 2010, 11:105.

48. Choi J, Kim KT, Jeon J, Lee YH: Fungal plant cell wall-degrading enzyme database: a platform for comparative and evolutionary genomics in fungi and Oomycetes. BMC Genomics 2013, 14(Suppl 5):S7.

49. Park J, Lee S, Choi J, Ahn K, Park B, Park J, Kang S, Lee YH: Fungal cytochrome P450 database. BMC Genomics 2008, 9:402.

50. Cheong K, Choi J, Choi J, Park J, Jang S, Lee YH: Eukaryotic DNAJ/K Database: A Comprehensive Phylogenomic Analysis Platform for the DNAJ/K Family. Genomics Inform 2013, 11(1):52-54.

51. Bailey $\mathrm{TL}$, Elkan C: Fitting a mixture model by expectation maximization to discover motifs in biopolymers. Proc Int Conf Intell Syst Mol Biol 1994, 2:28-36.

52. Hunter $S$, Jones $P$, Mitchell A, Apweiler R, Attwood TK, Bateman A, Bernard T, Binns D, Bork P, Burge $S$, de Castro E, Coggill P, Corbett M, Das U, Daugherty L, Duquenne L, Finn RD, Fraser M, Gough J, Haft D, Hulo N, Kahn D, Kelly E, Letunic I, Lonsdale D, Lopez R, Madera M, Maslen J, McAnulla C, McDowall J, et al: InterPro in 2011: new developments in the family and domain prediction database. Nucleic Acids Res 2012, 40(D1): D306-D312.

53. Roy K, Chanfreau GF: Chapter Ten - The Diverse Functions of Fungal RNase III Enzymes in RNA Metabolism. In The Enzymes. Volume 31. Academic Press; Guillaume FC, Fuyuhiko T 2012:213-235.
54. Hu Y, Stenlid J, Elfstrand M, Olson A: Evolution of RNA interference proteins dicer and argonaute in Basidiomycota. Mycologia 2013, 105(6):1489-1498.

55. Xu Z, Hao BL: CVTree update: a newly designed phylogenetic study platform using composition vectors and whole genomes. Nucleic Acids Res 2009, 37(W1):W174-W178.

56. Wang $\mathrm{H}, \mathrm{Xu} \mathrm{Z}$, Gao L, Hao BL: A fungal phylogeny based on 82 complete genomes using the composition vector method. BMC Evol Biol 2009 9:195.

57. Zuo G, Xu Z, Yu H, Hao B: Jackknife and bootstrap tests of the composition vector trees. Genomics Proteomics Bioinformatics 2010, 8(4):262-267.

58. Felsenstein J: PHYLIP (Phylogeny Inference Package) version 3.6. Distributed by the author Department of Genome Sciences, University of Washington, Seattle; 2005.

59. Tamura K, Stecher G, Peterson D, Filipski A, Kumar S: MEGA6: Molecular Evolutionary Genetics Analysis Version 6.0. Mol Biol Evol 2013, 30(12):2725-2729.

60. Chen K, Durand D, Farach-Colton M: NOTUNG: A program for dating gene duplications and optimizing gene family trees. J Comput Biol 2000, 7(34):429-447.

61. Wallace IM, O'Sullivan O, Higgins DG, Notredame C: M-Coffee: combining multiple sequence alignment methods with T-Coffee. Nucleic Acids Res 2006, 34(6):1692-1699.

62. Crooks GE, Hon G, Chandonia JM, Brenner SE: WebLogo: A sequence logo generator. Genome Res 2004, 14(6):1188-1190.

doi:10.1186/1471-2164-15-S9-S14

Cite this article as: Choi et al:: funRNA: a fungi-centered genomics platform for genes encoding key components of RNAi. BMC Genomics 2014 15(Suppl 9):S14.

\section{Submit your next manuscript to BioMed Central and take full advantage of:}

- Convenient online submission

- Thorough peer review

- No space constraints or color figure charges

- Immediate publication on acceptance

- Inclusion in PubMed, CAS, Scopus and Google Scholar

- Research which is freely available for redistribution

Submit your manuscript at www.biomedcentral.com/submit
C) Biomed Central 